\title{
Inhibition of NAMPT sensitizes MOLT4 leukemia cells for etoposide treatment through the SIRT2-p53 pathway
}

Theresa Grohmann ${ }^{\mathrm{a}}$, Melanie Penke ${ }^{\mathrm{a}}$, Stefanie Petzold-Quinque ${ }^{\mathrm{a}}$, Susanne Schuster ${ }^{\mathrm{a}}$, Sandy Richter ${ }^{\mathrm{a}}$, Wieland Kiess ${ }^{\mathrm{a}}$, Antje Garten ${ }^{\mathrm{a}, \mathrm{b}, *}$

${ }^{a}$ Hospital for Children \& Adolescents, Center for Pediatric Research Leipzig, University of Leipzig, Germany

${ }^{\mathrm{b}}$ University of Birmingham, Institute of Metabolism and Systems Research (IMSR), Birmingham, UK

Abstract

NAMPT (Nicotinamide phosphoribosyltransferase) catalyses the rate-limiting step in the NAD biosynthesis from nicotinamide and thereby regulates the activity of NAD-dependent enzymes. Cancer cells are highly dependent on NAD for energy and DNA repair processes and are assumed to be more susceptible to an inhibition of NAD synthesis than nontransformed cells. We aimed to investigate whether or not inhibition of NAMPT with its specific inhibitor FK866 can sensitize leukemia cells for chemotherapeutic agents.

NAMPT protein abundance, enzymatic activity and NAD concentrations were significantly higher in Jurkat and Molt-4 leukemia cell lines compared to normal peripheral blood mononuclear cells. Combination of eto- poside and FK866 caused increased cell death in leukemia cell lines compared to etoposide alone. Etoposide decreased protein abundance of NAD-dependent deacetylases SIRTUIN1. After combining etoposide and FK866 treatment SIRTUIN2 was further decreased and accumulation and acetylation of the downstream target p53 was further enhanced in MOLT4 cells. Concomitantly, protein abundance of p21 and cleaved BAX was increased.

Targeting NAMPT could be a novel therapeutic strategy to enhance the efficacy of chemotherapeutic agents such as etoposide against leukemia. 


\section{Introduction}

Leukemia is the most common cancer diagnosed in children and represents approximately $30 \%$ of cancer diagnoses among children younger than 15 years [1]. The main treatment for childhood leukemia is chemotherapy [2]. Children with high-risk leukemia or relapse gen- erally receive more intense and aggressive chemotherapy, e.g. treat- ment with etoposide [3,4]. Although chemotherapy is successful in up to $90 \%$ of pediatric patients with acute lymphoblastic leukemia, the toxicity of chemotherapy is a common cause of morbidity and mortality in children during treatment and later in life [5]. New strategies to reduce doses of chemotherapeutics are therefore urgently needed.

Cancer cells are characterized by metabolic adaptations such as a high nicotinamide adenine dinucleotide (NAD) turnover rate due to increased proliferation, DNA repair and metabolism [6,7]. Nicotina- mide phosphoribosyltransferase (NAMPT) is the key enzyme of the NAD salvage pathway from nicotinamide and thus a regulator of the intracellular NAD pool. NAMPT was shown to be overexpressed in different types of cancer, among them hematologic malignancies such as leukemia [8] and lymphomas [9]. Given that, NAMPT may be crucial for maintaining cellular NAD levels in cancer cells to facilitate cancer proliferation and survival. It is therefore a potential therapeutic target for the treatment of cancer.

SIRTUIN1 (SIRT1) and SIRT2 are NAD-dependent enzymes that are overexpressed in leukemia and could play a causal role in leukemia development Dan et al., 2012; Kozako et al., 2012; Wang et al., 2011b. Furthermore, these two central SIRTs play an important role in tumor metabolism by deacetylating p53 and therefore regulating apoptosis [10,11].

FK866 (APO866) is a specific inhibitor for NAMPT [12]. Treatment with FK866 reduced intracellular NAD levels leading to apoptosis and reduced cell proliferation in leukemia cell lines with functional tumor suppressor p53. This effect was mediated by increased acetylation of p53 at lysine 382 with subsequently increased expression of p21 and BAX. In contrast, leukemia cell lines containing nonfunctional p53 were relatively unaffected by FK866 treatment [13]. Furthermore, FK866 had minor toxic effects on normal hematopoietic progenitor cells [14]. Although FK866 was relatively well tolerated in humans and advanced to phase II clinical trials, it did not demonstrate sufficient tumor se- lectivity to achieve clinical success as single agent [15].

However, combining NAD depletion with chemotherapeutic agents might enhance their therapeutic efficacy [13]. In neuroblastoma cells, the effects of etoposide on DNA damage were potentiated by FK866 treatment, whereas the effect of FK866 on cytosolic NAD depletion was potentiated by etoposide [16]. In neuroendocrine tumors, the NAMPT inhibitor GMX1778 enhanced the efficacy of 177Lu-DOTATATE treat- ment and induced a prolonged antitumor response [17].

The aim of this study is to test the hypothesis that NAMPT inhibition using the specific inhibitor FK866 makes leukemia cells more suscep- tible for chemotherapeutic agents and to elucidate underlying signal- ling pathways.

\section{Materials \& methods}

\subsection{Material}

Cell culture media and supplements were obtained from PAA (Cölbe, Germany) or Invitrogen (Karlsruhe, Germany). FK866 and ni- cotinamide mononucleotide $(\mathrm{NMN})$ were obtained from Sigma Aldrich (Munich, Germany). Etoposide was purchased from Merck Millipore (Darmstadt, Germany). Primary antibodies were obtained from Cell Signaling (CST, Beverly, MA, USA) and Millipore. Secondary antibodies were purchased from DAKO (Hamburg, Germany).

\subsection{Cell culture}

Jurkat and Molt-4 T-ALL cell lines were purchased from Leibniz Institute DSMZ (German Collection of Microorganisms and Cell Cultures). The cell lines were cultured in RPMI 1640 medium with $2 \mathrm{mmol} / \mathrm{L}$ glutamine and 10\% fetal bovine serum (FBS) for Jurkat cells and 20\% FBS for Molt-4 cells. All cells were grown at $37{ }^{\circ} \mathrm{C}$ in a hu- midified atmosphere of $95 \%$ air and $5 \% \mathrm{CO}_{2}$. Cell number was mea- sured using a hemacytometer after trypan blue staining.

\subsection{PBMC isolation}

Peripheral blood mononuclear cells (PBMCs) were isolated from anonymised blood buffy coats of healthy donors purchased from the University Hospital Leipzig Blood Bank. Buffy coats were diluted in lysis buffer $(155 \mathrm{mM} \mathrm{NH} 4 \mathrm{Cl} ; 10 \mathrm{mM}$ KHCO3; $0.1 \mathrm{mM}$ Na-EDTA; pH 7.29), gently mixed and kept on ice for $10 \mathrm{~min}$. After centrifugation, the supernatant was removed carefully and the process was repeated until the pellet appeared clear.

\subsection{Cell treatments}

Etoposide was dissolved in DMSO to generate a stock solution of $42.5 \mathrm{mM}$. FK866 was dissolved in DMSO to create a stock solution of $10 \mathrm{mM}$. NMN was dissolved in the appropriate medium for a stock so- lution of $100 \mathrm{mM}$. Jurkat and Molt-4 cells were treated with the in- dicated concentrations of etoposide, indicated concentrations of FK866 or NMN [500 $\mu \mathrm{M}]$ either alone or in combination for $24 \mathrm{~h}$.

\subsection{Measurement of cell viability and cell death}

To investigate the effects of the chemotherapeutic agents on pro- liferation and cell viability, Cell Proliferation Reagent WST-1 (Roche, Grenzach-Wyhlen, Germany) was used according to manufacturer's instructions. 40,000 cells/well were seeded in a 96-well plate and cultured as described above.

To evaluate the effects of etoposide the number of dead cells was measured by flow cytometry using propidium iodide (PI) (Calbiochem, Dan Diego, CA, USA). 800,000 cells/well were seeded in a 6-well plate and cultured as described above. Cells were harvested and washed with ice-cold PBS. The cell pellet was re-suspended in $100 \mu \mathrm{L}$ of PBS with $0.5 \%$ FBS. Cell suspension was transferred into round bottom tubes. $2 \mu \mathrm{L}$ of PI were added to the cell suspension, followed by gentle vor- texing. Samples were incubated for $10 \mathrm{~min}$ on ice in darkness and analysed using a Beckton-Dickinson FACS LSRII. For each sample, 10,000 cells were counted. $\mathrm{PI}^{+}$cells were considered dead.

\subsection{Protein extraction and Western blot analyses}

Cells were lysed in modified RIPA buffer as previously described [18]. Protein concentration was determined using Pierce BCA protein assay (Thermo Scientific) and equal amounts of protein were separated by SDS-PAGE and transferred to nitrocellulose membranes using a semi-dry transfer apparatus. Next, membranes were blocked in 5\% non- fat dry milk in TBS buffer containing $0.1 \%$ Tween 20 . Primary anti- bodies used for immunoblotting included antiNAMPT (clone OMNI

379) (Cayman Chemical, Ann Arbor, MI, USA), anti-Caspase-3, anti- PARP, anti-SIRT1, anti-SIRT2, anti-Ac-p53, anti-p53, anti-p21, anti-Bax (Cell Signaling, Beverly, MA, USA) and anti-GAPDH (Merck Millipore, Schwalbach, Germany). Appropriate secondary antibodies were pur- chased from DAKO (Hamburg, 
Germany). Detection of proteins was carried out using Luminata Classico Western HRP Substrate (Merck Millipore) or Amersham ECL Prime Western Blotting Detection Reagent (GE Healthcare).

\subsection{NAMPT enzymatic activity}

NAMPT activity was measured by the conversion of ${ }^{14} \mathrm{C}$-labeled nicotinamide to ${ }^{14} \mathrm{C}$-NMN using a method previously described [19]. Radioactivity of ${ }^{14} \mathrm{C}$-NMN was quantified in a liquid scintillation counter (Wallac 1409 DSA, PerkinElmer). $1 \times 10^{6}$ cells were seeded in dishes $\left(152 \mathrm{~cm}^{2}\right)$ and $\mathrm{cultured}$ as described above. NAMPT activity (counts per minute, $\mathrm{cpm}$ ) was normalized to total protein concentration as measured by BCA protein assay.

\subsection{NAD measurement}

Concentrations of total NAD from whole-cell extracts were quanti- fied by HPLC analysis by reversed-phase HPLC using a Chromaster Purospher STAR RP-18 endcapped $3 \mu \mathrm{m}$ Hibar RT 150-3 HPLC column (Merck). 800,000 cells/well were seeded in a 6-well plate and cultured as described above. Cells were harvested and pellet was re-suspended in $100 \mu \mathrm{L} 1 \mathrm{M}$ perchloric acid. After a 10-min incubation period on ice samples were centrifuged and the supernatant was neutralized with $3 \mathrm{M}$ potassium carbonate. After repeated centrifugation samples were loaded onto the column as previously described [18].

The cell pellet of each sample was resuspended in $100 \mu \mathrm{L} 2 \%$ SDS,

shaken for $10 \mathrm{~min}$ at $99^{\circ} \mathrm{C}$ and centrifuged for $5 \mathrm{~min}$ at 20,000 $\mathrm{g}$ and then used for protein determination (BCA Assay, Pierce Thermo Scientific). The NAD concentration of each sample was referred to the corresponding total protein amount of the sample.

\subsection{Statistical analyses}

Data are presented as mean \pm SEM. Data were analysed for sta- tistical significance by either unpaired Student's $t$-test or one-way analysis of variance (ANOVA) followed by Bonferroni post hoc test. All analyses were performed using GraphPad Prism 6 software (GraphPad Software, Inc., San Diego, CA, USA). ImageJ 1.41 was used for densi- tometric analysis (NIH, USA). p $<0.05$ was considered significant compared to the referred control. 
A
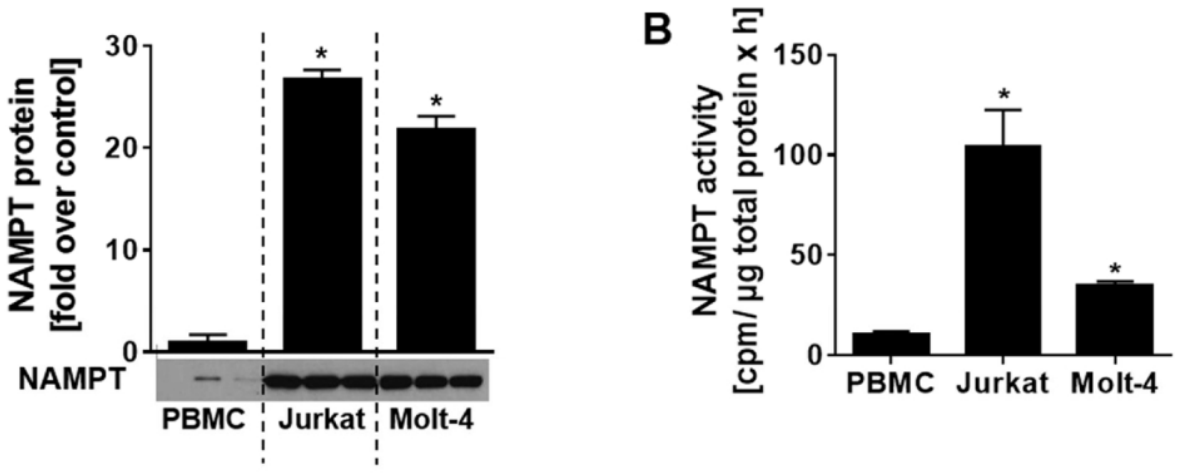

Fig. 1. NAMPT protein abundance, activity and NAD levels are higher in leukemia cell lines. NAMPT protein abundance (A), NAMPT activity (B) and NAD levels (C) were measured in PBMCs, Jurkat cells and Molt- 4 cells. Western Blots show results of 3 independent experiments and lanes were analyzed densito- metrically. Data are represented as mean \pm SEM and statistical analysis was per- formed using one-way ANOVA and Bonferroni post hoc test $(* \mathrm{p}<0.05$ compared to PBMCs). 2-column fitting image.

C

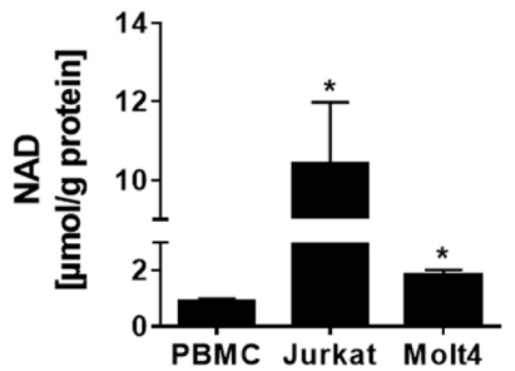

3. Results

\subsection{Cancer cell lines have higher NAMPT protein abundance and} activity

We compared the leukemia cell lines Jurkat (mutated p53) and Molt4 (wildtype p53) [20] with peripheral blood mononuclear cells (PBMCs) from healthy donors in regard to NAMPT protein abundance, NAMPT activity and NAD levels. Jurkat and Molt-4 cancer cell lines showed higher NAMPT protein abundance (25-fold in Jurkat cells, 21 - fold in Molt-4 cells) compared to PBMCs (Fig. 1A). Furthermore, NAMPT activity $(94.1 \pm 18.2$ $\mathrm{cpm} / \mu \mathrm{g}$ total protein $\mathrm{x} \mathrm{h}$ in Jurkat cells;

$24.6 \pm 2.6 \mathrm{cpm} / \mu \mathrm{g}$ total protein $\mathrm{x} \mathrm{h}$ in Molt- 4 cells) (Fig. 1B) and NAD levels were elevated in cancer cell lines $(9.5 \pm 1.6 \mu \mathrm{mol} \mathrm{NAD} / \mathrm{g}$ pro- tein in Jurkat cells, $0.9 \pm 0.2 \mu \mathrm{mol} \mathrm{NAD} / \mathrm{g}$ protein in Molt-4 cells) compared to PBMCs (Fig. 1C).

\subsection{FK866 decreases viability, NAMPT activity and NAD levels}

We tested the sensitivity of Jurkat and Molt-4 cell lines to inhibition of NAMPT using the specific inhibitor FK866. Incubation of cells with FK866[10 nM] for $24 \mathrm{~h}$ reduced viability $(-59.5 \pm 3.3 \%$ in Jurkat cells, $-46.1 \pm 4.6 \%$ in Molt-4 cells) (Fig. 2A,B)(Supporting Informa- tion 1 A,B). NAMPT activity was decreased in both cells lines sig-nificantly at a concentration of $1 \mathrm{nM} \mathrm{FK866}$ and was almost completely blocked after using a concentration of $10 \mathrm{nM}$ FK866 in both cell lines. (Fig. 2C,D).NAD concentrations were lowered (by $80 \%$ in Jurkat and Molt-4 cells) (Fig. 2 E,F)(Supporting Information 1C,D) compared to control cells. The enzymatic product of NAMPT, NMN [500 $\mu$ M], was able to rescue viability (Fig. 2A,B) and NAD levels (Fig. 2E,F) of both Jurkat and Molt-4 cells, whereas it had no effect alone.

\subsection{FK866 increases etoposide-induced cell death}

To evaluate whether NAMPT inhibition could enhance the effects of chemotherapeutic agents, Jurkat and Molt- 4 cells were incubated with the anti-leukemia drugs etoposide (eto), methotrexate ( $\mathrm{mtx}$ ) and dox- orubicin (doxo) alone or in combination with FK866 for $24 \mathrm{~h}$. Etoposide was able to induce cell death $(4.0$-fold $\pm 0.6[10 \mu \mathrm{M}])$ in Jurkat cells compared to control cells (Fig. 3A). Molt-4 cells were more sensitive to etoposide, which induced 5.6-fold $\pm 1.0[5 \mu \mathrm{M}]$ cell death (Fig. 3B). FK866 treatment alone did not induce cell death in both cell lines after $24 \mathrm{~h}$. However, the combination of etoposide and FK866 increased the number of dead cells compared to etoposide treatment alone (5.4- fold \pm 1.4 in Jurkat cells, 1.8fold \pm 0.1 in Molt- 4 cells) (Fig. 3A,B). NMN was able to reverse the FK866-induced effects. Methotrexate (mtx) and doxorubicin (doxo) only induced cell death in Molt- 4 cells $(+17.8 \% \pm 2.0 \%$ and $65.8 \% \pm 8.2 \%$, respectively). Combining mtx or doxo with FK866 did not increase cell death in Jurkat or Molt-4 cells (Supporting Information 2).

Etoposide treatment induced cleavage and activation of caspase- 3 and its downstream target poly (ADP-ribose) polymerase (PARP)-1, but this cleavage was not further increased by the addition of FK866 to etoposide (Fig. 3C, D). A combination of etoposide and FK866 did not alter cell cycle distribution after $24 \mathrm{~h}$ (Supporting Information 3).

\subsection{Etoposide-induced downregulation of SIRT2 is enhanced by NAD depletion}

Next, we aimed to determine whether the increased cell death provoked by etoposide and FK866 co-treatment could be caused by a further decrease in NAD levels. The NAD depletion induced by FK866 was not further enhanced by etoposide (Supporting Information 4). NAMPT protein abundance was not changed. However, etoposide treatment was able to decrease the amount of NAD-dependent deace- tylases SIRT1 and SIRT2 dosedependently in p53 wildtype Molt-4 cells. After etoposide treatment of NADdepleted cells SIRT2 was even further decreased (Fig. 4A). The acetylation and activation of the SIRT1 as well as SIRT2 target p53 was enhanced after cotreatment of etoposide with FK866, which was associated with an increased abundance of its downstream target p21 (Fig. 4B). Additionally, etoposide treatment induced cleavage of $\mathrm{p} 53$ downstream target BAX which was further enhanced by the combination with FK866. (Fig. 4B). Jurkat cells which harbour a mutated p53 did not show p53 acetylation or altered levels of p21 or cleaved bax (Supporting Information 5).

\section{Discussion}

Cancer cells have a high NAD turnover rate due to their increased proliferation, DNA repair, cell cycle progression and metabolism [6,7]. We therefore tested the efficacy of NAD depletion to sensitize leukemia cells for chemotherapeutic treatment with the aim to provide novel treatment options with reduced doses and consequently less adverse 

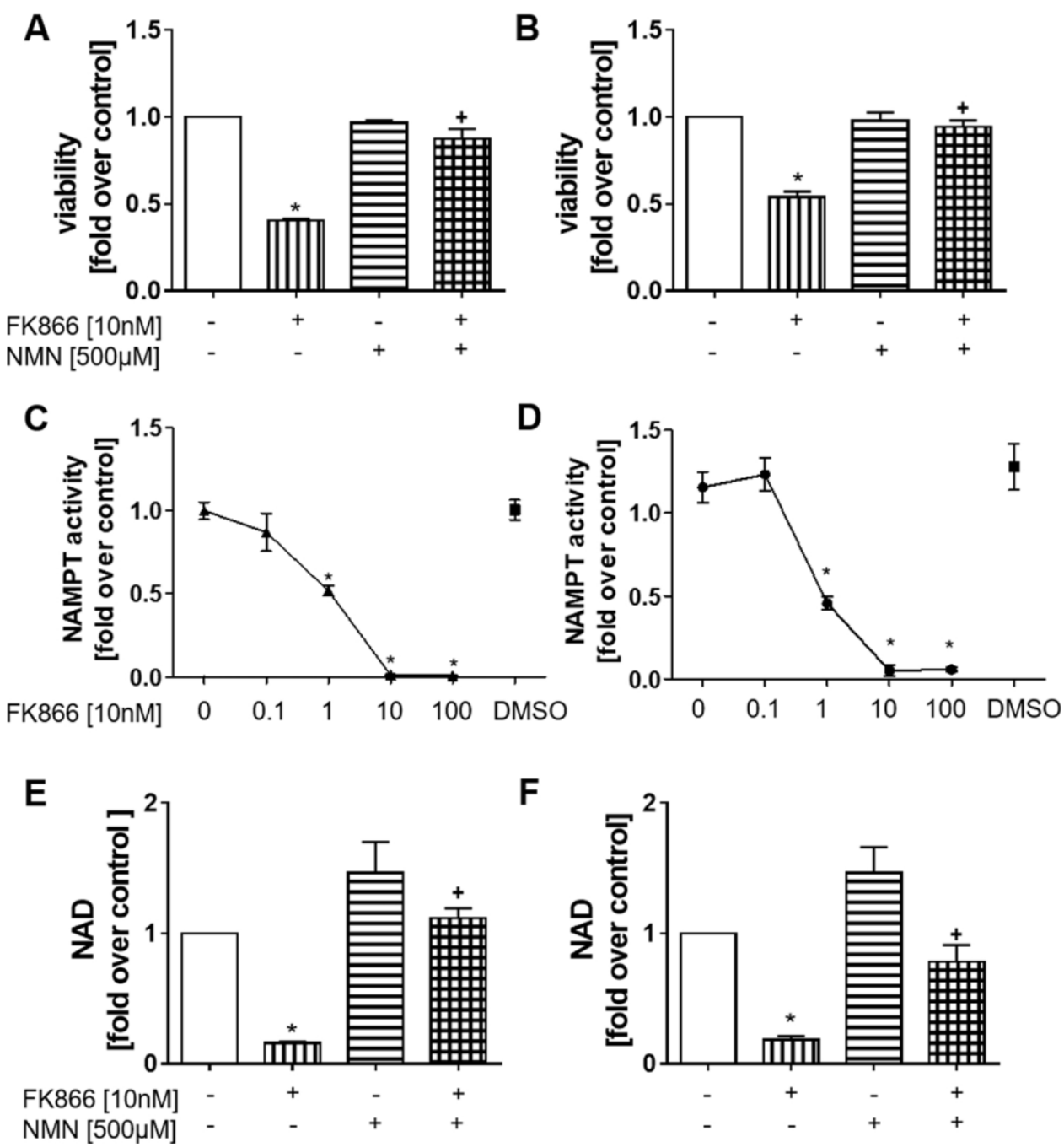

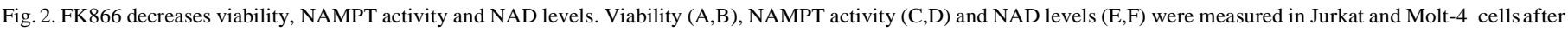

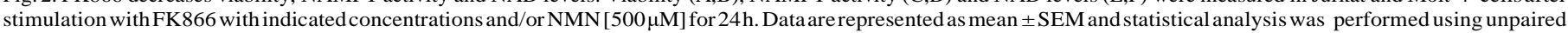
Student's t-test or one-way ANOVA with Bonferroni post hoc test $(* p<0.05$ compared to control; $+p<0.05$ compared to FK866). 2- column fitting image.

effects for patients.

The NAD salvage enzyme NAMPT was shown to be overexpressed in hematologic malignancies $[8,9,21]$. We could confirm these findings and furthermore showed that basal NAMPT activity and NAD levels were also increased in leukemia cells in comparison to PBMCs from healthy volunteers.

We used FK866 (APO866) to specifically target NAMPT [12]. Thakur et al. proposed that leukemia cell lines containing nonfunc- tional p53 were relatively unaffected by FK866 [13], whereas FK866 was able to induce cell death in p53-deficient hepatocarcinoma cells [22]. In our study, FK866 decreased viability, NAMPT activity and consequently depleted NAD levels in both p53 mutated (Jurkat) and p53 wildtype (Molt-4) cells.

Given the influence of NAD depletion on regulators of cellular survival and stress responses, such as PARPs and sirtuins, we postulated that FK866 could synergize with chemotherapeutic agents that target the same pathways. Travelli et al. showed that the etoposide-specific effect of inducing DNA damage was potentiated by FK866 [16]. Con- trary to their findings, however, we did not see an additive effect on total cellular NAD depletion (Supporting information 4). We could show that inhibition of NAMPT with FK866 in combination with etoposide led to increased cell death compared to etoposide alone. Interestingly, this effect was not mediated by the caspase cascade. This result stands in contrast to a study performed in HEK293 cells, where a knockdown of NAMPT made cells more sensitive to etoposide treatment and levels of cleaved caspase- 3 were increased, whereas the over- expression of NAMPT led to a resistance of cells to etoposide and re- duced levels of cleaved caspase 3 [23]. However, apoptosis, autophagy as well as necrosis were proposed as mechanisms of cell death induction by FK866 [6,14,24] or combination treatments $[16,21,25]$. The lack of apoptosis induction by FK866 could also be explained by the shorter treatment period we used compared to other studies [13,22].

SIRT1 and SIRT2 are overexpressed in leukemia cells, both are in- volved in disease development and drug resistance [26-28]. In our study, combining etoposide with FK866 decreased SIRT2 protein abundance in Molt-4 cells more than etoposide alone. Possible me- chanisms leading to selective SIRT2 depletion could involve the up regulation of microRNAs targeting SIRT2 [29], as has been reported for the effects of etoposide on SIRT1 protein [30].

P53 is a target of both SIRT1 and SIRT2 [10,11]. Blocking SIRT1 activity was shown to increase acetylation of p53 at lysine 382 in combination with DNA damaging agents $[31,32]$. In the p53 wildtype 
A

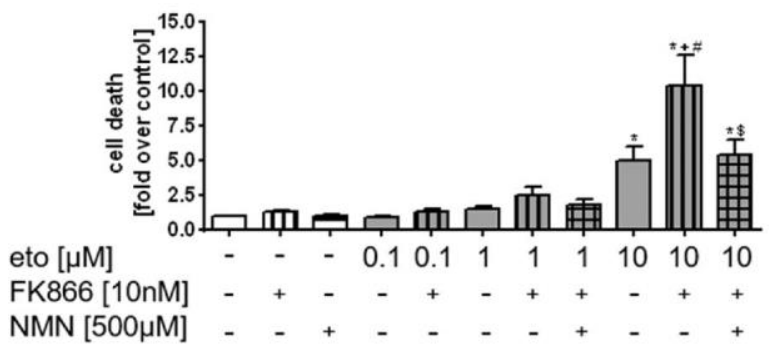

C
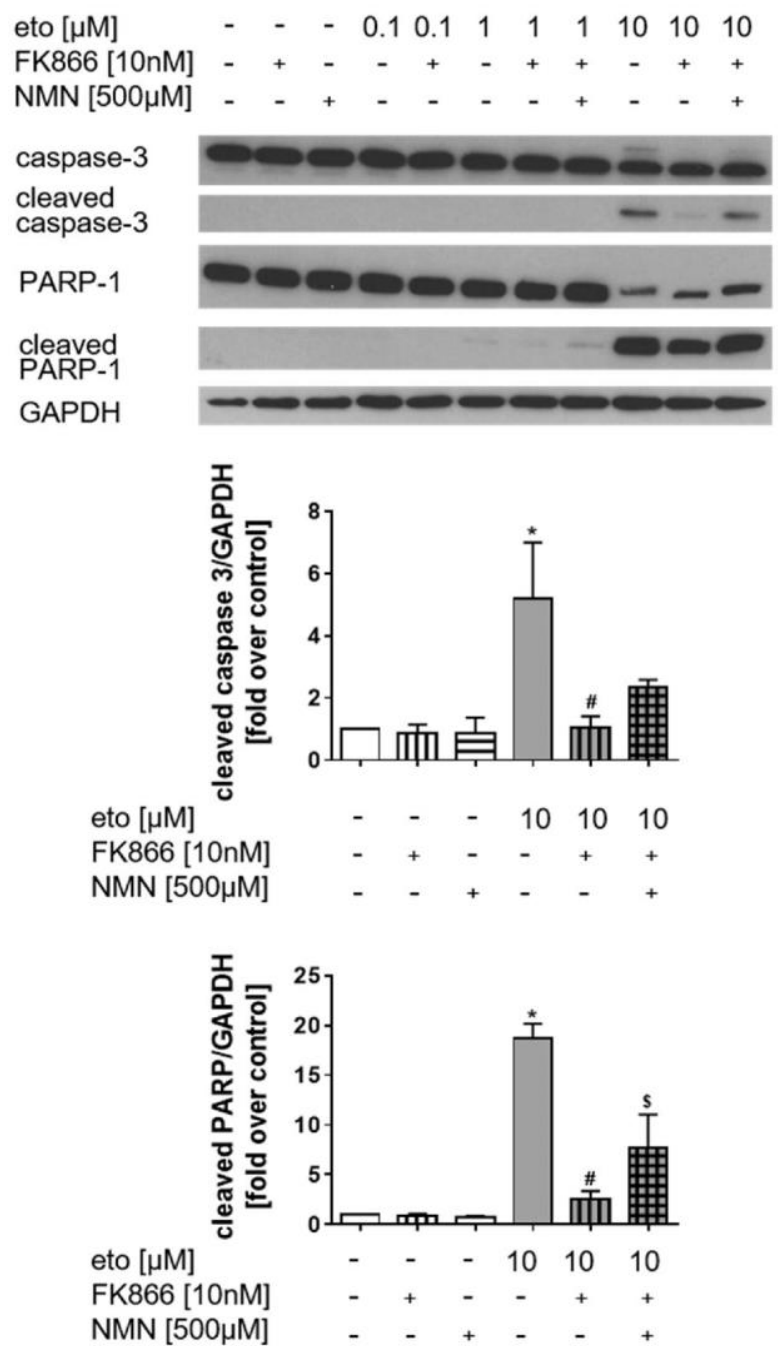

B

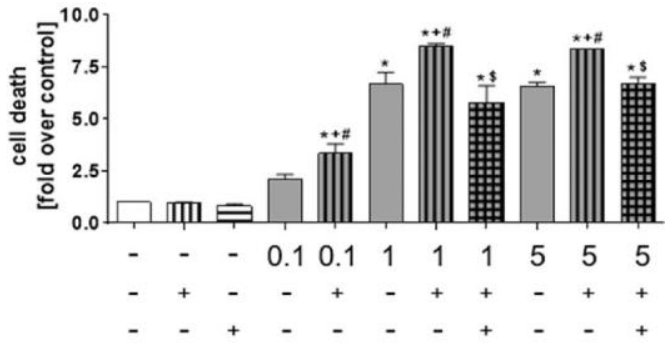

D
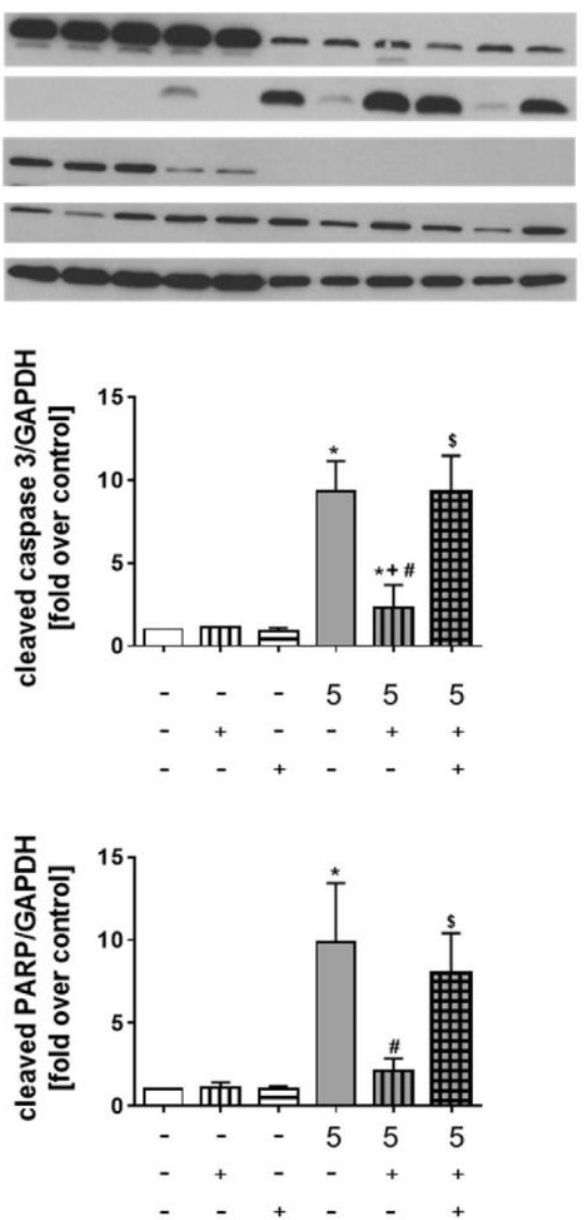

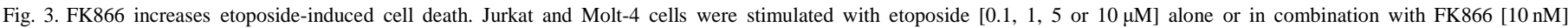
and/or NMN $[500 \mu \mathrm{M}]$ for $24 \mathrm{~h}$. Cell death was measured by flow cytometry using propidium iodide (A,B). Cleaved and full-lenght caspase-3 and

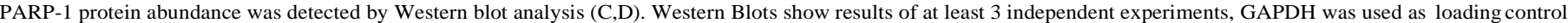

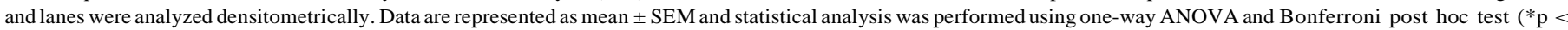
0.05 compared to control; $+\mathrm{p}<0.05$ compared to FK866 alone; \#p < 0.05 compared to etoposide [eto]; \$ compared to eto+FK866). 2-column fitting image. 
A
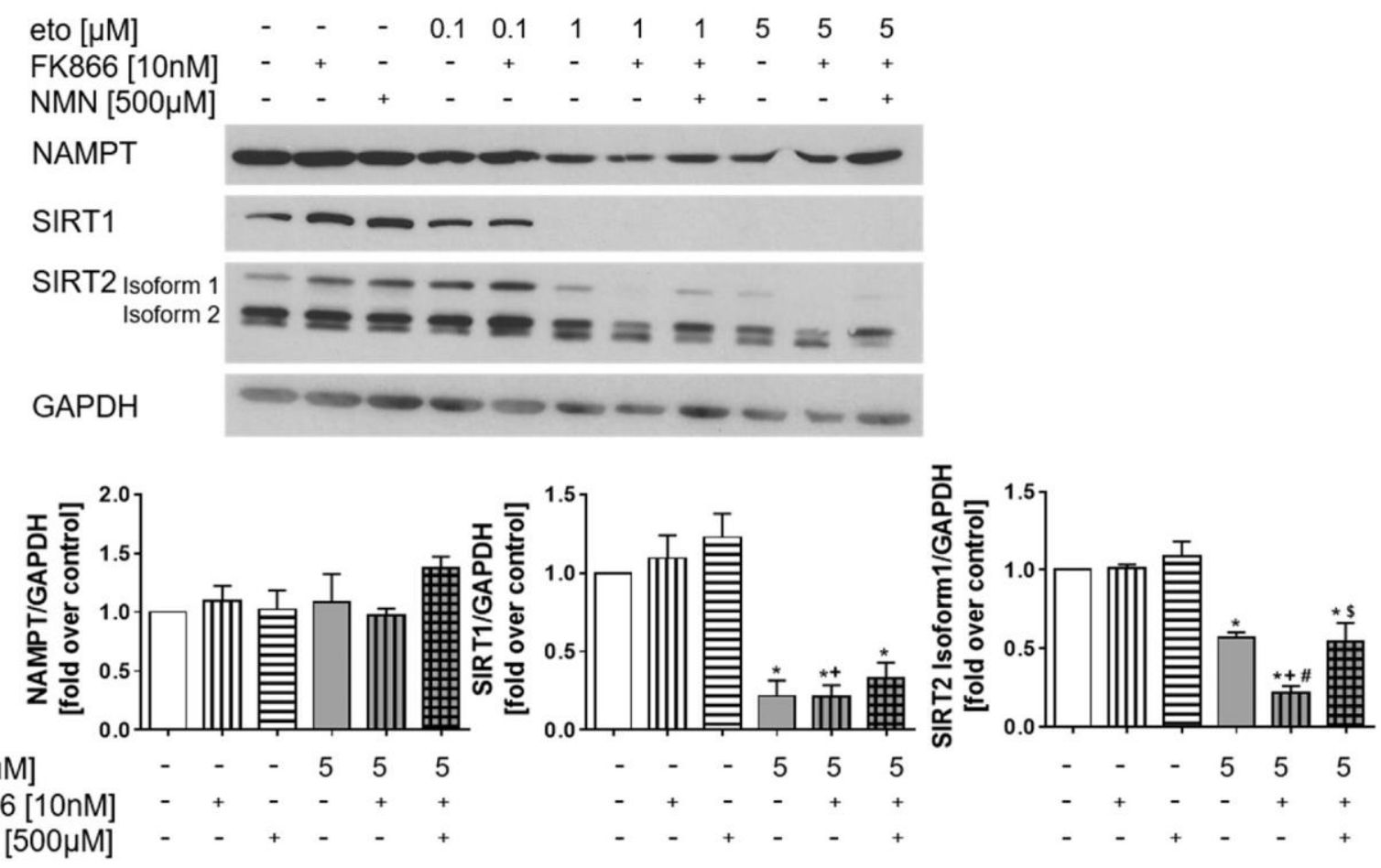

B

$\begin{array}{lllllll}\text { eto }[\mu M] & - & - & - & 5 & 5 & 5 \\ \text { FK866 [10nM] } & - & + & - & - & + & + \\ \text { NMN }[500 \mu M] & - & - & + & - & - & +\end{array}$
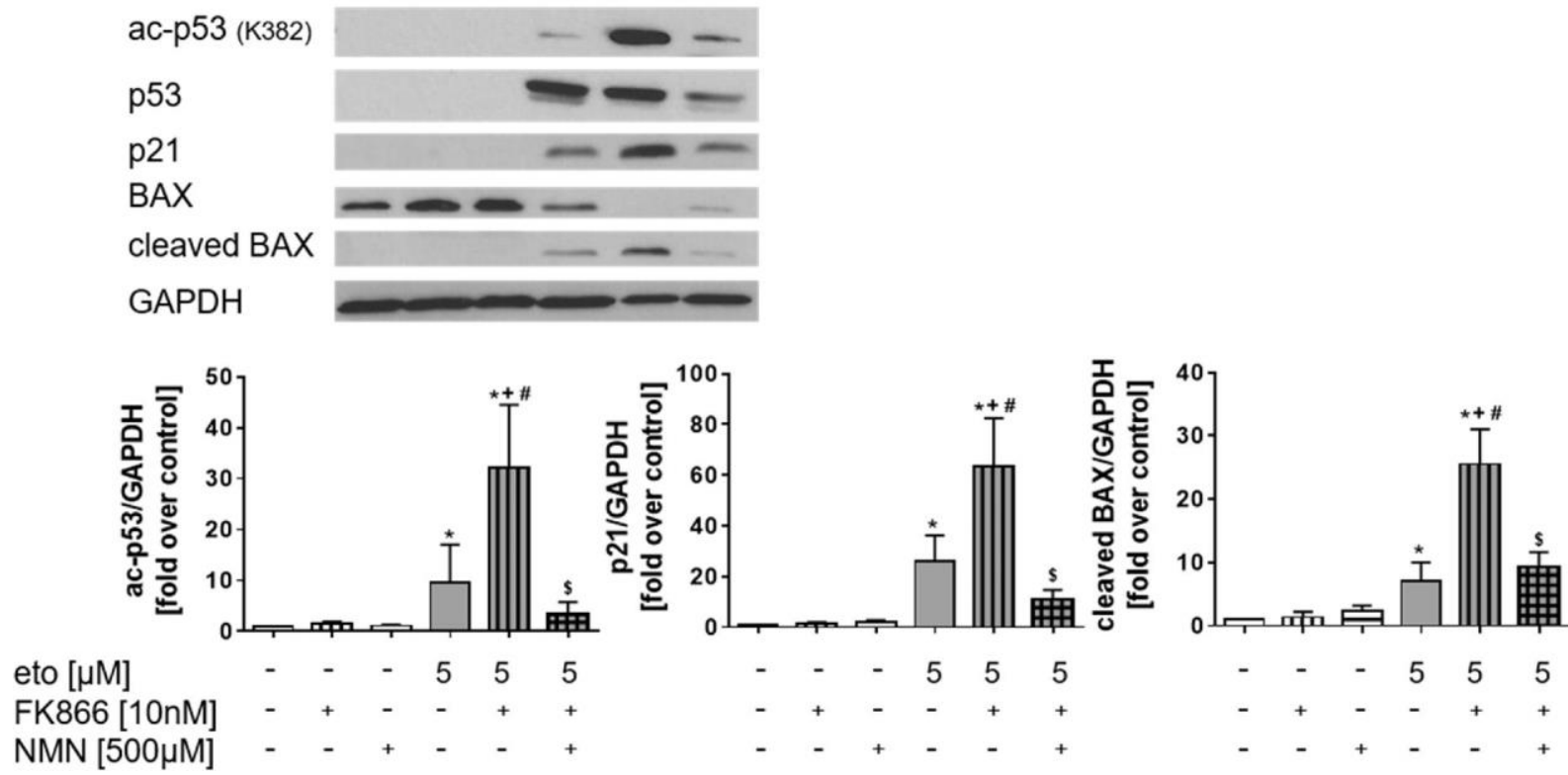

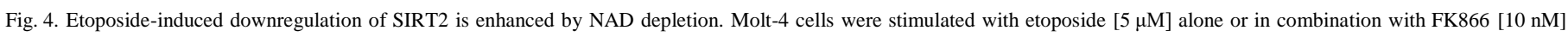
and/or NMN [500 $\mu \mathrm{M}$ ] for $24 \mathrm{~h}$. NAMPT, SIRT1 and SIRT2 (A), acetyl-p53, p53, p21 and BAX (B) protein abundance was analysed by Western blot.

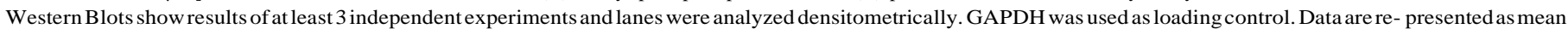

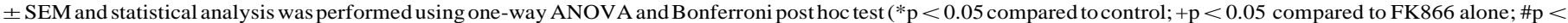
0.05 compared to etoposide [eto]; \$ compared to eto + FK866). 2-column fitting image.

cell line Molt-4, we found an accumulation of p53 after treatment with etoposide [33]. Furthermore, the combined effect of FK866 and eto- poside on SIRT2 was mirrored by an even stronger induction of p53 acetylation, higher expression of p53 downstream target p21 and cleavage of BAX. The cleavage of BAX during apoptosis was shown to increase the intrinsic cytotoxic properties of this proapoptotic factor [34]. This activation of p53-p21 signalling, as well as the increased cell death seen after co-treatment with etoposide and FK866, were 
reversible by supplementation with $\mathrm{NMN}$, which confirmed the effects to be specific for NAD depletion [35-37]. In Jurkat cells, which harbour a mutated p53, we did not detect p53 dependent changes in p21 or cleaved bax and no reduction in SIRT2 levels. A failure of p21 induction upon etoposide exposure has been shown before [38]. Potentially, cell death in Jurkat cells could be induced via c-Myc. SIRT2 inhibits c-Myc ubiquitination and degradation supporting tumorigenesis. Vice versa, reduced SIRT2 activity due to NAD depletion leads to cell death by c- Myc ubiquitination and degradation [39]. We therefore conclude that there are at least two pathways mediating the increased cell death seen upon combined treatment with etoposide and FK866 and that p53 is dispensable for cell death induction in this scenario.

\section{Conclusion}

Combined treatment with the NAMPT inhibitor FK866 potentiates the effects of chemotherapeutic agent etoposide and leads to a strong induction of cell death in vitro, via downregulation of SIRT2 and acti- vation of the p53p21 pathway in the p53 competent Molt- 4 cells. Targeting NAD salvage may be a useful strategy for chemo-sensitisation with the possibility to increase the efficacy of chemotherapeutic agents in the treatment of childhood leukemia. Additionally, SIRT2 inhibitors may represent interesting therapeutic options for p53 wildtype leu- kemia.

\section{Funding}

We thank "Stiftung Mitteldeutsche Kinderkrebsforschung" for financial support. The funder had no role in study design, data collection and analysis, decision to publish, or preparation of the manuscript.

\section{Conflict of interest}

All authors have nothing to disclose.

\section{Authorship}

TG, SPQ, SS, MP, SR, WK and AG conceived experiments and de- signed the study. TG, SPQ and SR carried out experiments. TG analysed the data. TG, SPQ, SS, MP, SR, WK and AG interpreted data and drafted the article. TG wrote the paper. All authors revised it critically for important intellectual content and had final approval of the submitted and published version.

\section{Acknowledgement}

We thank the Research Academy Leipzig and the Integrated Research Training Group "Obesity Mechanisms" (CRC 1052) for sup- port. This work has received funding from the European Union's Horizon 2020 research and innovation programme under the Marie Sklodowska-Curie grant agreement No 705869 (to A.G.) and from Deutsche Forschungsgemeinschaft/Collaborative Research Centre SFB1052 (to A.G. and W.K.).

\section{Appendix A. Supplementary data}

Supplementary data associated with this article can be found, in the online version, at https://doi.org/10.1016/j.leukres.2018.04.004.

\section{References}

[1] M. Belson, B. Kingsley, A. Holmes, Risk factors for acute leukemia in children: a review, Environ. Health Perspect. 115 (2007) 138-145.

[2] S.L. Cooper, P.A. Brown, Treatment of pediatric acute lymphoblastic leukemia, Pediatr. Clin. North Am. 62 (2015) 61-73.

[3] D. Bhojwani, S.C. Howard, C.-H. Pui, High-risk childhood acute lymphoblastic leukemia, Clin. Lymphoma Myeloma 9 (Suppl. (3)) (2009) S222-30.

[4] F. Locatelli, A.M. Testi, M.E. Bernardo, C. Rizzari, A. Bertaina, P. Merli, A. Pession, E. Giraldi, R. Parasole, W. Barberi, et al., Clofarabine, cyclophosphamide and etoposide as single-course re-induction therapy for children with refractory/multiple relapsed acute lymphoblastic leukaemia, Br. J. Haematol. 147 (2009) 371-378.

[5] G. Gervasini, J.M. Vagace, Impact of genetic polymorphisms on chemotherapy toxicity in childhood acute lymphoblastic leukemia, Front. Genet. 3 (2012) 249

[6] M. Cea, A. Cagnetta, F. Patrone, A. Nencioni, M. Gobbi, K.C. Anderson, Intracellular $\mathrm{NAD}(+)$ depletion induces autophagic death in multiple myeloma cells, Autophagy 9 (2013) $410-412$

[7] A. Chiarugi, C. Dölle, R. Felici, M. Ziegler, The NAD metabolome - a key determinant of cancer cell biology, Nat. Rev. Cancer 12 (2012) 741-752.

[8] V. Audrito, S. Serra, D. Brusa, F. Mazzola, F. Arruga, T. Vaisitti, M. Coscia, R. Maffei, D. Rossi, T. Wang, et al., Extracellular nicotinamide phosphoribosyltransferase (NAMPT) promotes M2 macrophage polarization in chronic lymphocytic leukemia, Blood 125 (2015) $111-123$

[9] U.H. Olesen, N. Hastrup, M. Sehested, Expression patterns of nicotinamide phosphoribosyltransferase and nicotinic acid phosphoribosyltransferase in human ma- lignan lymphomas, APMIS 119 (2011) 296-303.

[10] J. Luo, A.Y. Nikolaev, S. Imai, D. Chen, F. Su, A. Shiloh, L. Guarente, W. Gu, Negative control of p 53 by Sir2 $\alpha$ promotes cell survival under stress, Cell 107 (2001)
$137-148$

[11] B. Peck, C.-Y. Chen, K.-K. Ho, P. Di Fruscia, S.S. Myatt, R.C. Coombes, M.J. Fuchter, C. D. Hsiao, E.W.-F. Lam, SIRT inhibitors induce cell death and p53 acetylation through targeting both SIRT1 and SIRT2, Mol. Cancer Ther. 9 (2010).

[12] J.A. Khan, X. Tao, L. Tong, Molecular basis for the inhibition of human NMPRTase, a novel target for anticancer agents, Nat. Struct. Mol. Biol. 13 (2006) 582-588.

[13] B.K. Thakur, T. Dittrich, P. Chandra, A. Becker, W. Kuehnau, J.-H. Klusmann, D. Reinhardt, K. Welte, Involvement of p 53 in the cytotoxic activity of the NAMPT inhibitor FK866 in myeloid leukemic cells, Int. J. Cancer 132 (2013) 766-774.

[14] A. Nahimana, A. Attinger, D. Aubry, P. Greaney, C. Ireson, A. Thougaard, V. Tjørnelund, J. Dawson, K.M. Dupuis, M.A. Duchosal, The NAD biosynthesis in- hibitor APO866 has potent antitumor activity against hematologic malignancies, Blood 113 (2009) 3276-3286

[15] A. von Heideman, A. Berglund, R. Larsson, P. Nygren, Safety and efficacy of NAD depleting cancer drugs: results of a phase I clinical trial of CHS 828 and overview of published data, Cancer Chemother. Pharmacol. 65 (2010) 1165-1172.

[16] C. Travelli, V. Drago, E. Maldi, N. Kaludercic, U. Galli, R. Boldorini, F. Di Lisa G.C. Tron, P.L. Canonico, A.A. Genazzani, Reciprocal potentiation of the anti- tumoral activities of FK866, an inhibitor of nicotinamide phosphoribosyl- transferase, and etoposide or cisplatin in neuroblastoma cells, J. Pharmacol. Exp. Ther. 338 (2011) 829840

[17] A.-K. Elf, P. Bernhardt, T. Hofving, Y. Arvidsson, E. Forssell-Aronsson, B. Wangberg, O. Nilsson, V. Johanson, NAMPT inhibitor GMX1778 enhances the efficacy of 177Lu-DOTATATE treatment of neuroendocrine tumors, J. Nucl. Med. 58 (2) (2017) 288-292.

[18] S. Schuster, M. Penke, T. Gorski, S. Petzold-Quinque, G. Damm, R. Gebhardt, W. Kiess, A. Garten, Resveratrol differentially regulates NAMPT and SIRT1 in hepatocarcinoma cells and primary human hepatocytes, PLoS One 9 (2014).

[19] A. Garten, S. Petzold, A. Barnikol-Oettler, A. Korner, W.E. Thasler, J. Kratzsch, W. Kiess, R. Gebhardt, Nicotinamide phosphoribosyltransferase (NAMPT/PBEF/ visfatin) is constitutively released from human hepatocytes, Biochem. Biophys. Res. Commun. 391 (2010) $376-381$

[20] J. Cheng, M. Haas, Frequent mutations in the p53 tumor suppressor gene in human leukemia T-cell lines, Mol. Cell. Biol. 10 (1990) 5502-5509.

[21] A. Cagnetta, I. Caffa, C. Acharya, D. Soncini, P. Acharya, S. Adamia, I. Pierri, M. Bergamaschi, A. Garuti, G. Fraternali, et al., APO866 increases antitumor ac- tivity of Cyclosporin-A by inducing mitochondrial and endoplasmic reticulum stress in leukemia cells, Clin. Cancer Res. 21 (2015)

[22] S. Schuster, M. Penke, T. Gorski, R. Gebhardt, T.S. Weiss, W. Kiess, A. Garten, FK866 induced NAMPT inhibition activates AMPK and downregulates mTOR sig-nalling in hepatocarcinoma cells, Biochem. Biophys. Res. Commun. 458 (2015) 334-340.

[23] H. Yang, T. Yang, J.A. Baur, E. Perez, T. Matsui, J.J. Carmona, D.W. Lamming, N.C. Souza-Pinto, V.A. Bohr, A. Rosenzweig, et al., Nutrient-sensitive mitochondrial NAD+ levels dictate cell survival, Cell 130 (2007) 1095-1107.

[24] I. Gehrke, E.D.J. Bouchard, S. Beiggi, A.G. Poeppl, J.B. Johnston, S.B. Gibson, V. Banerji, On-target effect of FK866, a nicotinamide phosphoribosyl transferase inhibitor, by apoptosis-mediated death in chronic lymphocytic leukemia cells, Clin. Cancer Res. 20 (2014) 4861-4872.

[25] A. Nahimana, D. Aubry, C.S. Breton, S.R. Majjigapu, B. Sordat, P. Vogel, M.A. Duchosal, The anti-lymphoma activity of APO866, an inhibitor of nicotina- mide adenine dinucleotide biosynthesis, is potentialized when used in combination with antiCD20 antibody, Leuk. Lymphoma 55 (2014) 2141-2150.

[26] A. Deng, Q. Ning, L. Zhou, Y. Liang, SIRT2 is an unfavorable prognostic biomarker in patients with acute myeloid leukemia, Sci. Rep. 6 (2016) 27694.

[27] L. Li, R. Bhatia, Role of SIRT1 in the growth and regulation of normal hematopoietic and leukemia stem cells, Curr. Opin. Hematol. 22 (2015) 1.

[28] H. Xu, Y. Li, L. Chen, C. Wang, Q. Wang, H. Zhang, Y. Lin, Q. Li, T. Pang, SIRT2 mediates multidrug resistance in acute myelogenous leukemia cells via ERK1/2 signaling pathway, Int. J. Oncol. 48 (2015) 613-623.

[29] S. Li, X. Lv, K. Zhai, R. Xu, Y. Zhang, S. Zhao, X. Qin, L. Yin, J. Lou, MicroRNA-7 inhibits neuronal apoptosis in a cellular Parkinson's disease model by targeting Bax and Sirt2, Am. J. Transl. Res. 8 (2016) 993-1004.

[30] Y.N. Fan, D. Meley, B. Pizer, V. Sée, B. Pizer, S. Clifford, M. Taylor, P. Northcott, A. Korshunov, M. Remke, et al., Mir-34a mimics are potential therapeutic agents for p53mutated and chemo-resistant brain tumour cells, PLoS One 9 (2014) e108514.

[31] V. Audrito, T. Vaisitti, D. Rossi, D. Gottardi, G. D’Arena, L. Laurenti, G. Gaidano, F. Malavasi, S. Deaglio, Nicotinamide blocks proliferation and induces apoptosis of chronic lymphocytic leukemia cells through activation of the p53/miR-34a/SIRT1 tumor suppressor network, Cancer Res. 71 (2011) 4473-4483.

[32] J.M. Solomon, R. Pasupuleti, L. Xu, T. McDonagh, R. Curtis, P.S. DiStefano, L.J. Huber, Inhibition of SIRT1 catalytic activity increases p53 acetylation but does not alter cell survival following DNA damage, Mol. Cell. Biol. 26 (2006) 28-38.

[33] V.M. Golubovskaya, W.G. Cance, Targeting the p53 pathway, Surg. Oncol. Clin. N. Am. 22 (2013) 747-764

[34] D.E. Wood, E.W. Newcomb, Cleavage of bax enhances its cell death function, Exp. Cell Res. 256 (2000) 375-382.

[35] J.M. Espindola-Netto, C.C.S. Chini, M. Tarragó, E. Wang, S. Dutta, K. Pal, D. Mukhopadhyay, M. Sola-Penna, E.N. Chini, Preclinical efficacy of the novel competitive NAMPT inhibitor STF-118804 in pancreatic cancer, Oncotarget $8(2017)$ 85054-85067.

[36] L. Lu, L. Tang, W. Wei, Y. Hong, H. Chen, W. Ying, S. Chen, Nicotinamide mononucleotide improves energy activity and survival rate in an in vitro model of Parkinson's disease, Exp. Ther. Med. 8 (2014) 943-950.

[37] J. Yoshino, K.F. Mills, M.J. Yoon, S. Imai, Nicotinamide mononucleotide, a key NAD + intermediate, treats the pathophysiology of diet- and age-induced diabetes in mice, Cell Metab. 14 (2011) 528-536.

[38] C. Davies, L.A. Hogarth, P.A. Dietrich, P.S. Bachmann, K.L. Mackenzie, A.G. Hall, R.B. Lock, p53-Independent epigenetic repression of the p21 WAF1 gene in T-cell acute lymphoblastic leukemia, J. Biol. Chem. 286 (2011) 37639-37650.

[39] H. Jing, J. Hu, B. He, Y.L. Negrón Abril, J. Stupinski, K. Weiser, M. Carbonaro, Y. L. Chiang, T. Southard, P. Giannakakou, et al., A SIRT2-selective inhibitor promotes c-Myc oncoprotein degradation and exhibits broad anticancer activity, Cancer Cell 29 (2016) 297310 